\title{
InGaN/GaN Distributed Feedback Laser Diodes with Surface Gratings and Sidewall Gratings
}

\author{
Zejia Deng ${ }^{1,2}$, Junze Li ${ }^{1,2, *}$, Mingle Liao ${ }^{1,2}$, Wuze Xie ${ }^{1,2}$ and Siyuan Luo ${ }^{1,2}$ \\ 1 Microsystems and Terahertz Research Center, China Academy of Engineering Physics, Chengdu 610200, \\ China; dengzejia@mtrc.ac.cn (Z.D.); liaomingle@mtrc.ac.cn (M.L.); xiewuze@mtrc.ac.cn (W.X.); \\ luosiyuan@mtrc.ac.cn (S.L.) \\ 2 Institute of Electronic Engineering, China Academy of Engineering Physics, Mianyang 621999, China \\ * Correspondence: lijunze@mtrc.ac.cn
}

Received: 15 September 2019; Accepted: 12 October 2019; Published: 14 October 2019

check for updates

\begin{abstract}
A variety of potential applications such as visible light communications require laser sources with a narrow linewidth and a single wavelength emission in the blue light region. The gallium nitride (GaN)-based distributed feedback laser diode (DFB-LD) is a promising light source that meets these requirements. Here, we present GaN DFB-LDs that share growth and fabrication processes and have surface gratings and sidewall gratings on the same epitaxial substrate, which makes LDs with different structures comparable. By electrical pulse pumping, single-peak emissions at 398.5 and $399.95 \mathrm{~nm}$ with a full width at half maximum (FWHM) of 0.32 and $0.23 \mathrm{~nm}$ were achieved, respectively. The surface and sidewall gratings were fabricated alongside the p-contact metal stripe by electrical beam lithography and inductively coupled plasma etching. DFB LDs with $2.5 \mu \mathrm{m}$ ridge width exhibit a smaller FWHM than those with 5 and $10 \mu \mathrm{m}$ ridge widths, indicating that the narrow ridge width is favorable for the narrowing of the line width of the DFB LD. The slope efficiency of the DFB LD with sidewall gratings is higher than that of surface grating DFB LDs with the same ridge width and period of gratings. Our experiment may provide a reliable and simple approach for optimizing gratings and GaN DFB-LDs.
\end{abstract}

Keywords: GaN laser diode; distributed feedback (DFB); surface gratings; sidewall gratings

\section{Introduction}

Gallium nitride (GaN) laser diodes (LDs) are potentially used in displays, medical application, visible light communications (VLC), etc [1-4]. Some applications require a single peak and narrow-linewidth laser source. Distributed feedback lasers diodes (DFB-LDs) with these advantages have attracted extensive concern in both academia and the industry. In optical atomic clocks, an extremely narrow-linewidth blue laser is required to aim at atomic cooling transition [5]. In medical diagnostics, the DFB LD is a promising light source for fluorescence spectroscopy, where the emission wavelength can be precisely targeted [6].

GaN-based DFB LDs have been achieved by buried gratings [7-9], sidewall gratings, and surface gratings [10-13]. In the beginning, the first order DFB LD was achieved by establishing the buried gratings [14]. Subsequently, the third and 39th DFB LDs were fabricated by etching sidewall gratings [15-17]. Additionally, the single longitudinal mode emissions of the 10th DFB LDs with surface gratings were realized under optical pumping and electrical impulse driving $[18,19]$. In contrast to burying gratings, DFB LDs with sidewall and surface gratings do not require high-cost and hard crystal regrowth processing, preventing the device from being damaged in the regrowth process $[20,21]$. Surface gratings and sidewall gratings are preferred for DFB LDs due to simpler fabrication processes. Though there have been varieties of DFB LDs fabricated using surface gratings or sidewall gratings, 
to the best of our knowledge, all of them, regardless of grating type, are achieved on different epitaxial wafers; there are no reports on GaN DFB-LDs that share growth and fabrication processes and have surface gratings and sidewall gratings on the same epitaxial substrate. Therefore, it is inevitable that there are distinct differences in the optical and electrical properties of DFB LDs with different kinds of grating structures. It is difficult to systematically compare and analyze the performance of DFB LDs with surface and sidewall gratings, because they are fabricated on diverse epitaxial wafers. Hence, in order to further understand the performance differences of DFB LDs with different structures, it is necessary to fabricate DFB LDs with different types of gratings on the same epitaxial substrate, which can provide some experimental evidences for the optimal design of DFB LDs.

In this work, the finite-difference-time-domain (FDTD) tool was used to analyze the influence of the width of the ridge and the types of gratings on the properties of DFB LDs. Based on the designed structure of LDs, DFB-LDs with surface and sidewall gratings of different periods and ridge widths were fabricated on the same epitaxial wafer. Fabry-Pérot (F-P) LDs with the same ridge widths were also fabricated on the same epi-wafer for comparison. The well-proportioned gratings were defined alongside the p-contact metal stripe by electrical beam lithography (EBL) and inductively coupled plasma (ICP) etching. The morphology characteristics of all the LDs were observed by a scanning electron microscope (SEM) with an FEI Nova NanoSEM 450. The electrical and optical characteristics of the DFB LDs, including the linewidth, slope efficiency, and threshold current, were measured and analyzed.

\section{Simulation and Fabrication}

According to the coupled mode theory, the coupling coefficient can be achieved approximately by the following equation [22]:

$$
\kappa=\frac{2\left(n_{2}-n_{1}\right)}{\lambda_{1}} \frac{\sin (\pi m \gamma)}{m}
$$

where $m$ is the grating order, $\lambda_{1}$ is the wavelength, $n_{1}$ is the effective modal index in the broad area including the sections of the ridge and grating, $n_{2}$ is the effective modal index in the section of the ridge, and $\gamma$ is the duty-cycle of gratings.

The effective modal index ( $n_{\text {eff }}$ ) of the waveguide in a GaN LD can be calculated using the finite-difference-time-domain (FDTD), where the epitaxial structure and device structure of the simulation can be found in the parameters displayed in the following sections. Generally, there is a decreasing trend in modal index with ridge width at a certain etch depth. Typical optical field distributions are given in Figure 1, with ridge widths of 2.5 and $5 \mu \mathrm{m}$. It is obvious that a reduction of the waveguide width restriction the optical field more, resulting in a decreasing in mode number.
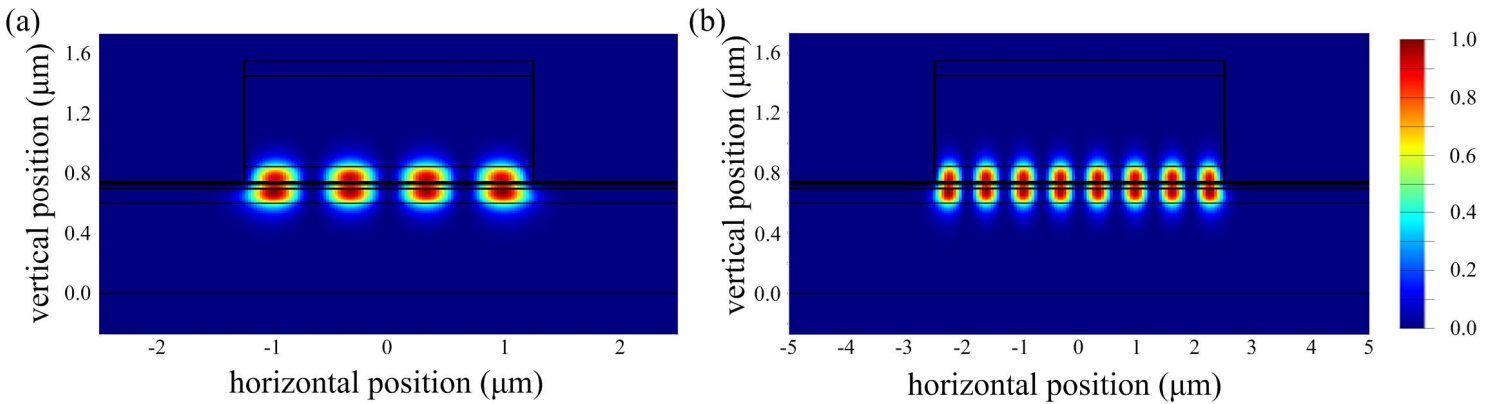

Figure 1. Optical field profiles for ridge widths of (a) $2.5 \mu \mathrm{m}$ and (b) $5 \mu \mathrm{m}$.

The refractive modal index increases with ridge width and gradually approaches the maximum value when the ridge width is relatively large, which is shown in Figure 2a. It is worthy pointing out that coupling length $k L$ has a great influence on the characteristics of the DFB LDs. Figure $2 b$ shows the coupling length $k L$ as a function of the ridge width when the etch depth was $500 \mathrm{~nm}$ and 
the cavity length was $600 \mu \mathrm{m}$. For the sidewall grating, the broad section including the ridge and grating had a width twice the size of the section of the ridge, whereas for the surface grating, the broad section possessed a fixed width of $80 \mu \mathrm{m}$. Obviously, $k L$ showed a decreasing trend as the ridge width increased. It must be pointed out that this calculation was based on the fundamental mode; in the high order, mode $k L$ had a relatively large value, leading to a moderate reflectivity. Since the surface gratings had a greater width (and thus refractive index) in the broad area for the same ridge width (compared with the sidewall gratings), they had a bigger refractive index difference $\Delta n=\left(n_{2}\right.$ $-n_{1}$ ). As demonstrated in Equation (1), the coupling length $k L$ was proportional to refractive index difference $\Delta \mathrm{n}$ when the order and duty ratio of the gratings were defined. As such, the $k L$ of the DFB LD with surface gratings was slightly higher than that of the one with sidewall gratings when the ridge widths of the two kinds of structures were identical.
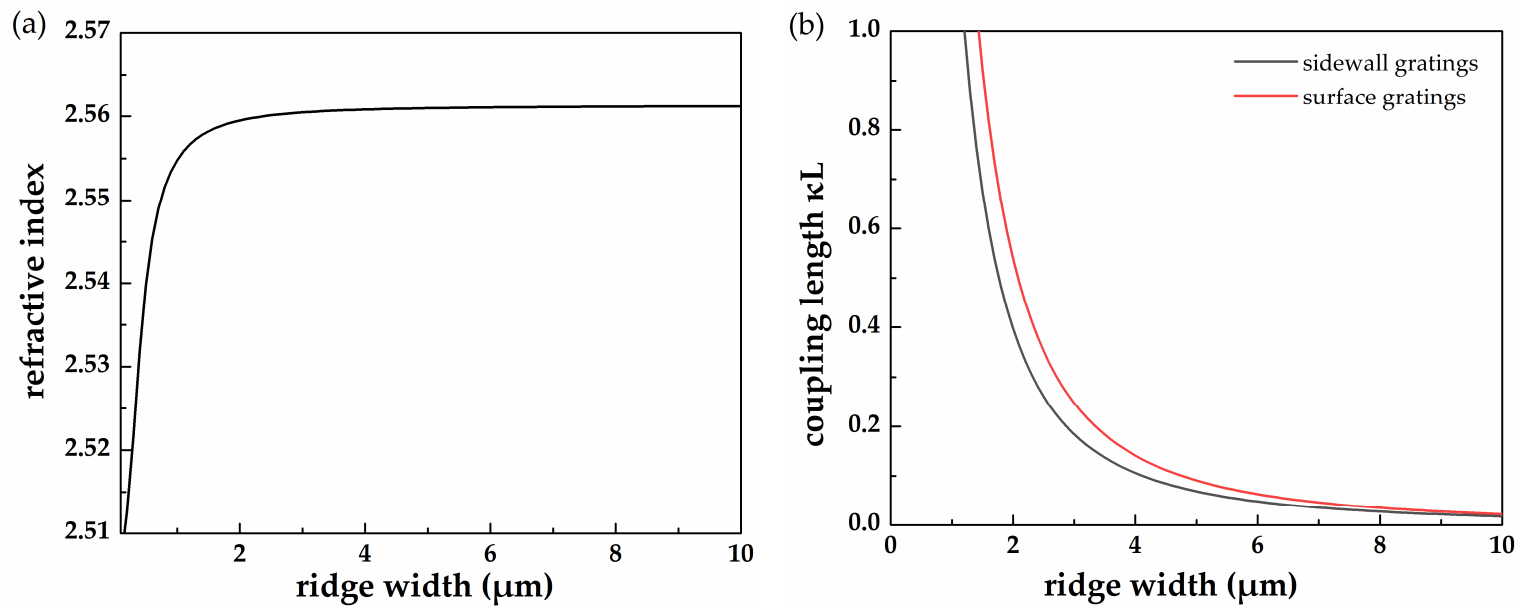

Figure 2. (a) Refractive index as a function of the ridge width. (b) Coupling length $k L$ as a function of the ridge width of the sidewall and surface gratings. Etching depth: $500 \mathrm{~nm}$.

According to the Bragg condition, $\Lambda=\lambda \mathrm{m} / 2 n_{\mathrm{eff}}$ and the characterization of the emission at around $403 \mathrm{~nm}$ of the F-P LDs fabricated on the epitaxial wafer, the periods of the 10th-order and 20th-order Bragg grating were calculated and found to be 824 and $1648 \mathrm{~nm}$, respectively. Another parameter for the gratings is the duty cycle $\gamma=(\Lambda-o) / \Lambda$, where $o$ denotes the width of the etched trench. A duty cycle of around 0.8 was adjusted for the gratings.

The designed complete structures of the DFB LDs with surface and sidewall gratings are depicted in Figure 3. It is worth noting that the surface gratings had a larger grating width than the sidewall grating and were much wider than the width of the ridge waveguide, while the width of the sidewall grating was closer to that of the ridge waveguide. The LDs were fabricated from the AlInGaN epi-structure composed of n-type and p-type epitaxial layers and InGaN/GaN multiple quantum wells (MQWs) designed for the emission of $403 \mathrm{~nm}$, including a lower cladding layer of $850 \mathrm{~nm}$ $\mathrm{Al}_{0.075} \mathrm{Ga}_{0.925} \mathrm{~N}: \mathrm{Si}$, a 60-nm-thick $\mathrm{In}_{0.03} \mathrm{Ga}_{0.97} \mathrm{~N}: \mathrm{Si}$ lower waveguide layer, a multiple quantum well with three 6.5- or 2-nm-thick undoped GaN quantum barriers and two $2.7 \mathrm{~nm} \mathrm{In}_{0.1} \mathrm{Ga}_{0.9} \mathrm{~N}$ wells, an upper waveguide layer of doped $60 \mathrm{~nm}$ InGaN:Mg, a $20 \mathrm{~nm} \mathrm{Al}_{0.13} \mathrm{G}_{0.87} \mathrm{~N}: \mathrm{Mg}$ electron blocking layer, a $450 \mathrm{~nm}$ $\mathrm{Al}_{0.05} \mathrm{Ga}_{0.95} \mathrm{~N}: \mathrm{Mg}$ upper cladding layer, and a $10 \mathrm{~nm} \mathrm{GaN}: \mathrm{Mg}$ subcontact layer. The F-P LDs and DFB LDs were fabricated on the same epi-wafer by the same manufacturing process except for the building of the surface and sidewall gratings of DFB LDs. The 2.5-, 5- and 10- $\mu \mathrm{m}$-wide Ni/Au p-contact stripes with a thickness of 10/30 nm were formed on the wafer by electron beam evaporation and a rapid thermal process. The 150-nm-thick $\mathrm{SiO}_{2}$ layer was deposited on the whole wafer by plasma-enhanced chemical vapor deposition (PECVD) as the hard mask of etching of gratings and ridge waveguides for DFB LDs. The 250-nm-thick positive polymethyl methacrylate (PMMA) resist was used to define the gratings with periods of 824 and $1648 \mathrm{~nm}$, the patterns of the gratings were constructed on both sides of the p-contact stripes and perpendicular to them with this method of electron beam lithography (EBL), 
the model of the EBL was Raith i-line plus with a write resolution of $7 \mathrm{~nm}$. The patterns of gratings and ridge waveguides were transferred to the epi-structure of the wafer through reactive ion etching based on $\mathrm{CHF}_{3} / \mathrm{SF}_{6}$ gas and the dry etching of inductively coupled plasma (ICP) using $\mathrm{Cl}_{2} / \mathrm{BCl}_{3}$ gas in succession. The $300 \mathrm{~nm}$-thick $\mathrm{SiO}_{2}$ insulating layer was formed on the wafer by PECVD, and the $2-, 4.5-$ and $8.5-\mu \mathrm{m}$-wide openings on the ridge were given shape by dry etching the $\mathrm{SiO}_{2}$ layer using $\mathrm{CHF}_{3} / \mathrm{SF}_{6}$ mixed gas before the $50 / 250 \mathrm{~nm}$ Ti/Au metal pad was deposited. The back side of the wafer was thinned and polished to a thickness of $120 \mu \mathrm{m}$, and then an n-contact Ti/Pt/Au metal layer with a thickness of 50/50/250 nm was deposited on it. The processed sample was cut into bars with a cavity length of $600 \mu \mathrm{m}$, and both front and back facets were uncoated.

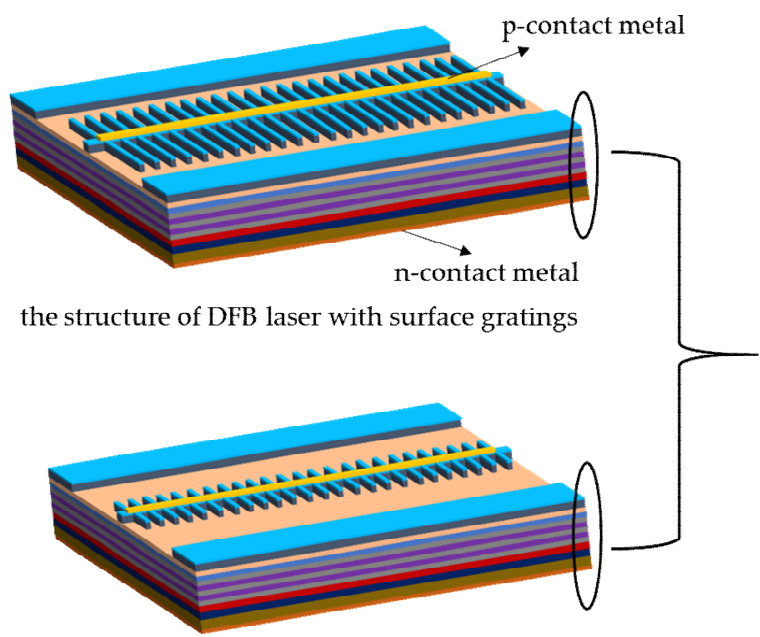

the structure of DFB laser with sidewall gratings

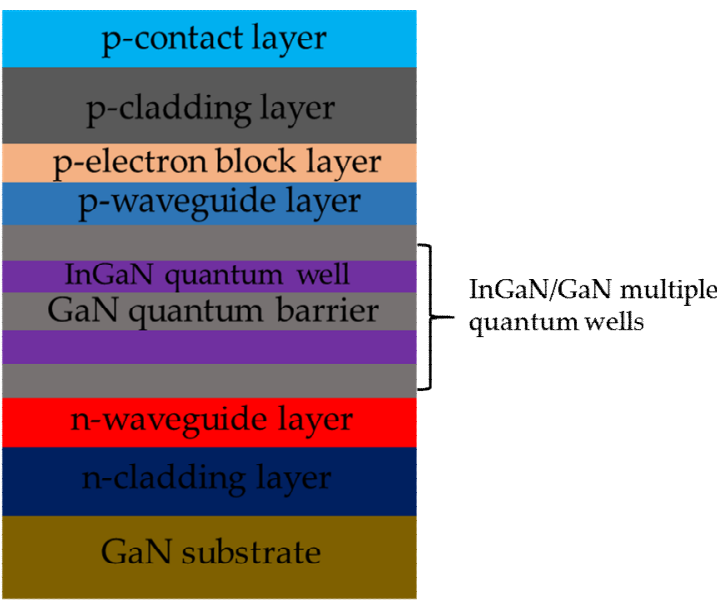

epitaxial structure

Figure 3. The structures of processed distributed feedback laser diodes (DFB-LDs) with surface gratings and sidewall gratings, as well as the diagrammatic drawing of the cross profile of the gallium nitride (GaN)-based epitaxial wafer.

\section{Results and Discussions}

\subsection{The DFB LDs with the Surface Gratings}

Table 1 compares the structure parameters of different manufactured LDs. Devices 1 and 4 are F-P LDs with ridge widths of 2.5 and $10 \mu \mathrm{m}$, respectively, that are compared with the fabricated DFB-LDs with surface gratings. Devices 2 and 3 have the same ridge width and width and duty ratio of surface gratings, but they have different grating periods of 824 and $1648 \mathrm{~nm}$. The same parameters also apply to Devices 5 and 6 except for the ridge width. All of them were fabricated from the same epitaxial wafer and shared the same processing.

Table 1. The structure parameters of the fabricated distributed feedback laser diodes (DFB-LDs) of surface gratings and Fabry-Pérot (F-P) LDs.

\begin{tabular}{ccccccc}
\hline Sample & Device 1 & Device 2 & Device 3 & Device 4 & Device 5 & Device 6 \\
\hline Ridge width & $2.5 \mu \mathrm{m}$ & $2.5 \mu \mathrm{m}$ & $2.5 \mu \mathrm{m}$ & $10 \mu \mathrm{m}$ & $10 \mu \mathrm{m}$ & $10 \mu \mathrm{m}$ \\
Width of grating of each side & - & $40 \mu \mathrm{m}$ & $40 \mu \mathrm{m}$ & - & $40 \mu \mathrm{m}$ & $40 \mu \mathrm{m}$ \\
Period of gratings & $\mathrm{z}$ & $824 \mathrm{~nm}$ & $1648 \mathrm{~nm}$ & - & $824 \mathrm{~nm}$ & $1648 \mathrm{~nm}$ \\
\hline Duty ratio of grating & - & $80 \%$ & $80 \%$ & - & $80 \%$ & $80 \%$ \\
\hline
\end{tabular}

The structures of the ridge and gratings of the DFB LDs with surface gratings were well formed, as shown in Figure 4. The high resolution SEM graph in Figure 4a shows a lateral view of the DFB LD with surface gratings, thus displaying the general structural features of the device. The actual 
parameters of the ridge and the grating of the processed DFB LD are shown in Figure $4 \mathrm{~b}$, which were close to the designed values shown in Table 1. Figure 4c presents the sectional feature of the surface gratings with a period of $1648 \mathrm{~nm}$. It can be concluded that the gratings have the desired quality and a depth of $481 \mathrm{~nm}$ close to the head of the upper waveguide layer, which means that they basically meet our design requirements.
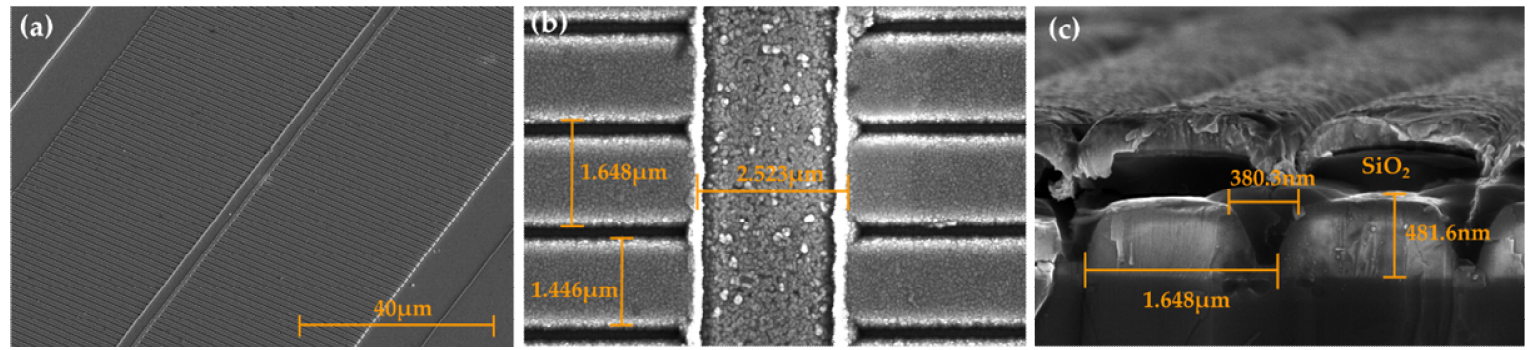

Figure 4. Scanning electron microscope (SEM) images of (a) an oblique view of the fabricated DFB laser diodes with surface gratings, (b) a top vision of the 20th order surface gratings alongside a metal stripe, and (c) a sectional structure of the surface gratings perpendicular to the facet of LDs.

The spectral measurements were conducted by a fiber spectrum analyzer (BIM6002, Brolight, Hangzhou, China) with a resolution of $0.16 \mathrm{~nm}$ under a pulsed operation of a $500 \mathrm{~ns}$ pulse length and $1 \mathrm{kHz}$ repetition frequency. Figure 5 shows the emission spectrum chart of the DFB LDs (Devices 1-6) with surface gratings and F-P LDs. All of them were operated at the impulsive condition of 1.2 times the threshold current by the fiber spectrometer. Since the emission peaks of those LDs had different intensities under certain test currents, the differences in the position of the bottom of the spectrums of LDs were caused by the normalization process of dividing by the different highest intensities. The emission peaks of Devices 1-6 were located at 402.13, 398.86, 398.50, 402.86, 400.50 and $400.86 \mathrm{~nm}$, respectively. Obviously, the DFB LDs had a shorter lasing wavelength than the F-P LDs with the same ridge width because of the interaction between gratings and multiple quantum wells. The offset of the 2 3 nm emission wavelength between the DFB and the F-P laser also confirms the modulation effect of the grating. The full width at half maxima (FWHM) corresponding to DFB LDs for Devices $2,3,5$, and 6 were $0.37,0.32,0.52$, and $0.50 \mathrm{~nm}$, respectively. We can conclude that the DFB LDs exhibited a narrower emission width and a single peak emission because of the modulation of the gratings, while the F-P LDs for Devices 1 and 4 had distinct multimode characteristics. Additionally, the FWHM of the emission of the DFB LD with the same ridge seems to have rarely been related to the period of the gratings, while the DFB LD with the $2.5 \mu \mathrm{m}$ ridge width had a lower FWHM than that with the $10 \mu \mathrm{m}$ ridge width. This can be partly explained by the fact that, as depicted in Figure 1 , the simultaneous oscillation of lateral modes sharing the same longitudinal mode number eventually results in a wider spectral width. In addition, according to the equation $L=\lambda m / 2 n_{\mathrm{eff}}-$ where $n_{\mathrm{eff}} \approx 2.5$ and $L=600 \mu \mathrm{m}$ - the expected free spectral range (FSR) of the longitudinal modes of the F-P laser could be approximately $0.05 \mathrm{~nm}$ at around $400 \mathrm{~nm}$. Therefore, the DFB LDs possibly showed multi-mode operation, but this phenomenon was not been confirmed because of the lack of the measurement condition of the high resolution spectrum and the lateral far field. 


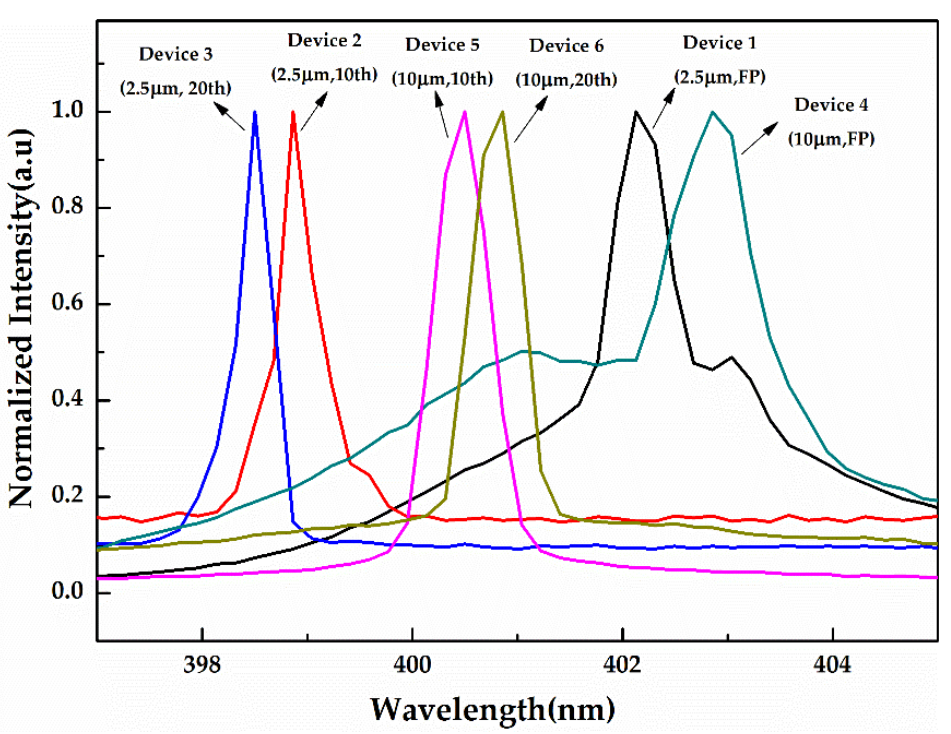

Figure 5. Emission spectrograms of fabricated laser diodes (Devices 1-6) driven by electrical impulses with a $500 \mathrm{~ns}$ pulse width and a $1 \mathrm{kHz}$ repetition rate.

\subsection{The DFB LDs with the Sidewall Gratings}

In addition, the DFB LDs with sidewall gratings were fabricated on the same epitaxial wafer, and they shared the same processing steps with the surface grating DFB LDs. The structure parameters of the fabricated LDs are shown in Table 2. Devices 7 and 8 had the same ridge width of $2.5 \mu \mathrm{m}$, gratings width of $1.25 \mu \mathrm{m}$, and $80 \%$ duty ratio of gratings, but they had different grating periods of 824 and $1648 \mathrm{~nm}$, respectively. The same parameters for DFB LDs with sidewall gratings except for the widths of the ridge and gratings were applied to Devices 10 and 11. Device 9 was the F-P LDs with a ridge width of $5 \mu \mathrm{m}$, which was in contrast with the DFB LDs for Devices 10 and 11.

Table 2. Structure parameters of the fabricated DFB LDs of sidewall gratings and F-P LDs.

\begin{tabular}{cccccc}
\hline Sample & Device 7 & Device 8 & Device 9 & Device 10 & Device 11 \\
\hline Ridge width & $2.5 \mu \mathrm{m}$ & $2.5 \mu \mathrm{m}$ & $5 \mu \mathrm{m}$ & $5 \mu \mathrm{m}$ & $5 \mu \mathrm{m}$ \\
Width of gratings of each side & $1.25 \mu \mathrm{m}$ & $1.25 \mu \mathrm{m}$ & - & $2.5 \mu \mathrm{m}$ & $2.5 \mu \mathrm{m}$ \\
Period of gratings & $824 \mathrm{~nm}$ & $1648 \mathrm{~nm}$ & - & $824 \mathrm{~nm}$ & $1648 \mathrm{~nm}$ \\
\hline Duty ratio of gratings & $80 \%$ & $80 \%$ & - & $80 \%$ & $80 \%$ \\
\hline
\end{tabular}

Figure 6 shows that the DFB LD with 20th order sidewall gratings was well fabricated. Figure 6a is the top view of the DFB LD with sidewall gratings, and it depicts the structure of ridge, sidewall gratings, double etching grooves, and the p-contact metal on the ridge. Figure $6 b, c$ shows the actual parameters of the ridge and gratings of the processed device, which are basically in conformity to the values in Table 2. Sidewall gratings with a period of $1648 \mathrm{~nm}$, a duty ratio of approximately $80 \%$, and a width of $1.25 \mu \mathrm{m}$ of each side alongside the ridge waveguide were observed. Since the corresponding etchings processes were under the same experimental condition, the surface and sidewall gratings had a similar etching depth and sectional morphology, as shown in Figure 4. The notches of the gratings had a tilt angle as a result of the ICP etch process, so the average duty ratio of the fabricated gratings was greater than $80 \%[23,24]$. Moreover, the simulation results showed that the influence of the relative large grating angle of $70^{\circ}$ could be ignored [25], offering a high enough reflectivity for the DFB LDs. 

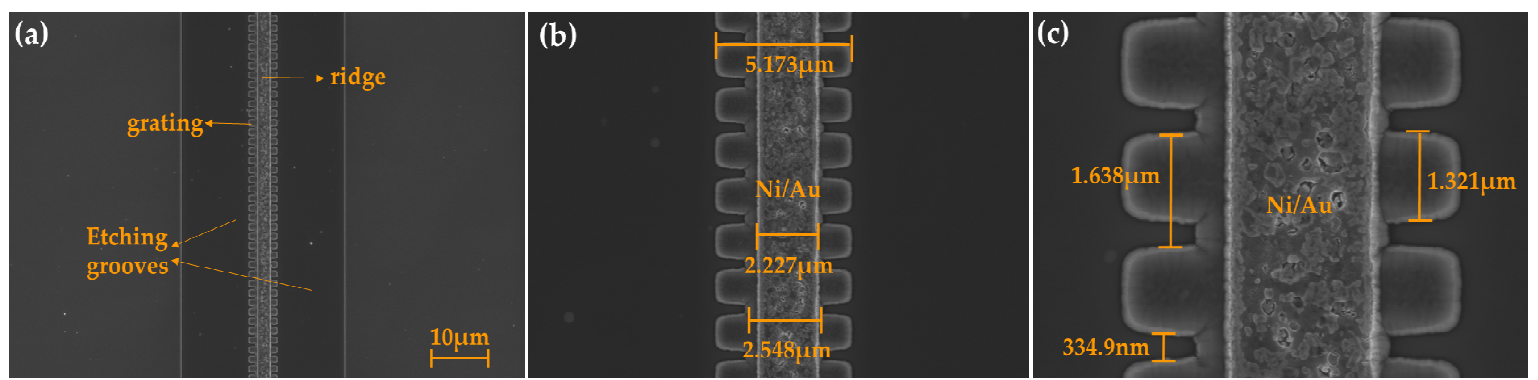

Figure 6. SEM images of a top view of the fabricated DFB laser diodes with 20th order sidewall gratings. (a) An overall view of the DFB LD with double etching grooves; (b) a low power and (c) a high power perspective of the ridge waveguide and the sidewall gratings along it.

The emission spectrum of the DFB LDs with sidewall gratings and the F-P LDs for Devices 7-11 can be seen in Figure 7, and the LDs were operated at a current around 20\% above threshold current. The emission peaks of the Devices 7-11 were 399.59, 399.95, 403.76, 401.4 and $401.77 \mathrm{~nm}$, respectively. The FWHMs of the DFB LDs for Devices 7, 8, 10, and 11 were $0.25,0.23,0.42$, and $0.48 \mathrm{~nm}$, respectively, which were slightly higher than the resolution of the fiber spectrometer. In the context of comparing the multimode morphology of F-P LDs with the ridge of $5 \mu \mathrm{m}$ during lasing, the emission spectrum of the DFB LDs showed a single peak because of the existence of the gratings. Similar to the previous results, the DFB LDs of sidewall gratings had a lower lasing wavelength than the F-P LDs with the same ridge width. In addition, the DFB LDs with a $2.5 \mu \mathrm{m}$ ridge width had a lower FWHM than those with the $5 \mu \mathrm{m}$ ridge width, thus indicating that a narrow ridge width is favorable for the narrowing of the linewidth of the DFB LD. According to the spectrum characteristics, we could speculate that the effect of grating periods on the line width of LDs is not very evident.

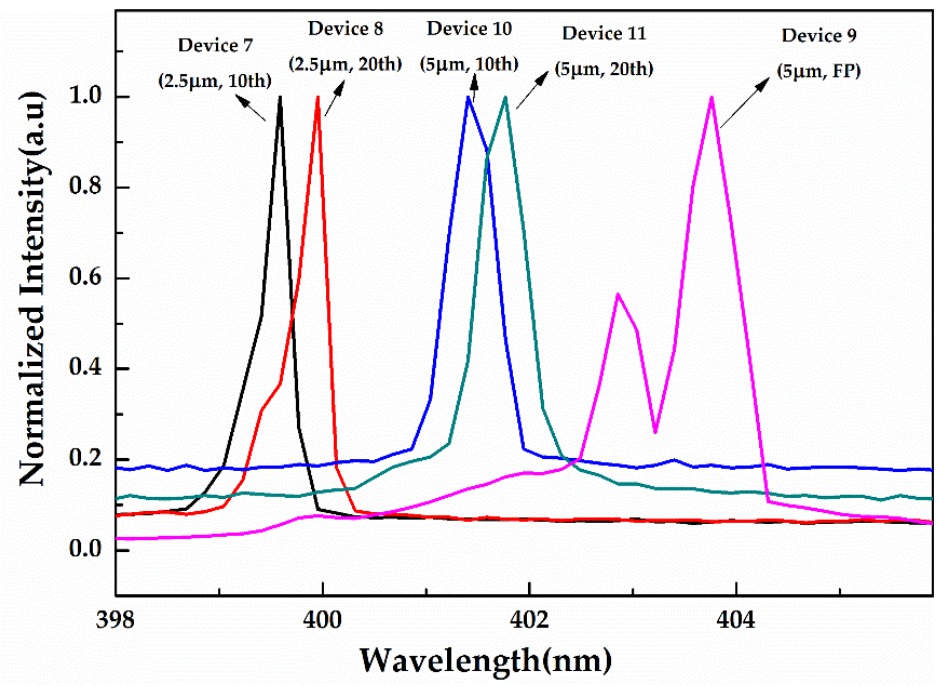

Figure 7. Emission spectrograms of processed laser diodes for Devices 7-11 driven by electrical impulses with a $500 \mathrm{~ns}$ pulse width and a $1 \mathrm{kHz}$ repetition rate.

\subsection{The Comparison of Properties of the DFB LDs with Surface and Sidewall Gratings}

Meanwhile, based on the good linewidth characteristics of DFB LDs with a ridge width of $2.5 \mu \mathrm{m}$, the characteristics of DFB LDs with sidewall and surface gratings and the F-P LD were compared in the case that their ridge widths were all $2.5 \mu \mathrm{m}$. The sidewall gratings need lower costs for writing a smaller area of patterns than surface gratings which use EBL. The light output power and voltage versus current $(\mathrm{L}-\mathrm{I}-\mathrm{V})$ characteristics of the LDs were tested under the pulsed driving condition with a pulse width of $1 \mu$ s and a pulse repetition frequency of $10 \mathrm{kHz}$ at room temperature to avoid generating excess heat. Figure 8 shows L-I-V characteristics of the DFB LDs and the F-P LDs with the same 
ridge width of $2.5 \mu \mathrm{m}$. The values of the threshold currents of Devices $1,2,3,7$, and 8 were between 450 and $560 \mathrm{~mA}$, and their slope efficiencies were approximately 0.24, 0.12, 0.17, 0.13, and 0.19 W/A, respectively. The deviations of the threshold current and slopes efficiency of the LDs with the same structures were less than $8 \%$ and $13 \%$, respectively. However, all of the F-P and DFB LDs tested showed comparatively high threshold currents and lower slope efficiencies, which was mainly deemed to owe to the performance of the epitaxial wafer. More intuitive and detailed results are shown in Table 3.

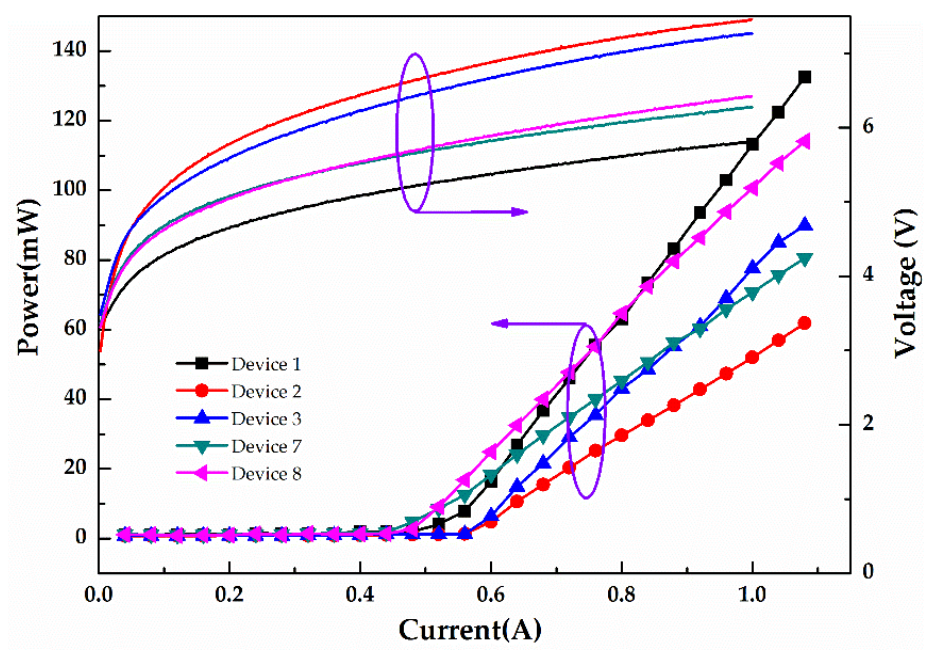

Figure 8. Light output power-current-voltage characteristics of Devices 1, 2, 3, 7, and 8 operated in pulsed mode with a $1 \mu$ s pulse width and a $10 \mathrm{kHz}$ repetition rate.

Table 3. Electrical properties of the fabricated DFB LDs and F-P LDs.

\begin{tabular}{cccccc}
\hline Sample & Device 1 & Device 2 & Device 3 & Device 7 & Device 8 \\
\hline Type of gratings & - & Surface & Surface & Sidewall & Sidewall \\
Order of gratings & - & 10 th & 20th & 10 th & 20 th \\
Threshold current & $(518 \pm 12) \mathrm{mA}$ & $(539 \pm 4) \mathrm{mA}$ & $(550 \pm 10) \mathrm{mA}$ & $(458 \pm 7) \mathrm{mA}$ & $(485 \pm 19) \mathrm{mA}$ \\
The slopes & $(235 \pm 15)$ & $(116 \pm 3)$ & $(172 \pm 11)$ & $(129 \pm 7)$ & $(189 \pm 6)$ \\
efficiency & $\mathrm{mW} / \mathrm{A}$ & $\mathrm{mW} / \mathrm{A}$ & $\mathrm{mW} / \mathrm{A}$ & $\mathrm{mW} / \mathrm{A}$ & $\mathrm{mW} / \mathrm{A}$ \\
FWHM & $1.96 \mathrm{~nm}$ & $0.37 \mathrm{~nm}$ & $0.32 \mathrm{~nm}$ & $0.25 \mathrm{~nm}$ & $0.23 \mathrm{~nm}$ \\
\hline
\end{tabular}

From the data in Figure 8 and Table 3, it can be seen that DFB LDs with the same ridge width and a lower grating period exhibited lower slope efficiency because of the higher value of $k L$ compared to those with high order gratings [26]. In addition, the slope efficiency of DFB LDs with sidewall gratings was slightly higher than that of the DFB LDs with surface gratings but smaller than F-P LDs because of the scatter losses and diffraction losses from the gratings. Besides, the FWHM of DFB LDs with sidewall gratings was slightly lower than that of DFB LDs with surface gratings, and this may have been due to the fact that the sidewall grating DFB LD had a narrower effective width in the whole structure compared to the surface grating of the DFB LD with the same ridge width. Given its smaller grating area, the sidewall grating DFB LD had fewer transverse modes, thus resulting in a smaller FWHM compared to the surface grating DFB LD. Additionally, the linewidth of the DFB LDs with a single peak emission showed a drastic reduction compared to that of the F-P LDs with multiple lasing peaks.

\section{Conclusions}

In conclusion, the GaN-based DFB LDs that integrated with the surface and sidewall gratings were investigated by the FDTD method. The surface and sidewall gratings alongside the p-contact metal stripe on the ridge waveguide were fabricated by EBL and ICP etching on the same epitaxial 
wafer. The DFB LD with the 20th order, $80 \%$ duty-cycle surface gratings showed a single wavelength emission at $398.86 \mathrm{~nm}$ with an FWHM of $0.32 \mathrm{~nm}$ under the electrical pulsed driving, and the DFB LD with the 20th order, $80 \%$ duty-cycle sidewall gratings obtained a peak emission at $399.95 \mathrm{~nm}$ with an FWHM of $0.23 \mathrm{~nm}$. Additionally, both of the DFB LDs showed a narrower linewidth compared to that of the F-P LDs. Moreover, the FWHM of the DFB LDs with the ridge width of $2.5 \mu \mathrm{m}$ was obviously lower than that of the DFB LDs using the same type of gratings with ridge widths of 5 or $10 \mu \mathrm{m}$, which indicates that the narrow ridge width was favorable for the narrowing of the linewidth. Furthermore, the sidewall grating DFB LDs possessed a slightly higher slope efficiency than that of the surface grating DFB LDs with the same ridge width and period of gratings. Given that, the DFB LD with sidewall grating required a lower fabrication cost and achieved better device performance compared to the surface grating DFB LDs, which makes it a better choice for these applications. In addition, in order to further improve the performance of the DFB LDs, the optimization of the structure of the gratings and the conduction of the cavity surface coating process are required to reduce threshold current and improve slope efficiency. Additionally, the side-mode suppression ratio and the high resolution spectral measurement are also required in future work.

Author Contributions: Conceptualization-Z.D. and J.L.; data curation-Z.D. and M.L.; methodology-Z.D. and M.L.; project administration-J.L.; supervision-J.L.; validation-W.X. and S.L.; writing original draft-Z.D.

Funding: This work was funded by Science Challenge Project (No. TZ2016003-2), National Natural Science Foundation of China (No. 61804140), and National Key R\&D Program of China (No. 2017YFB0403103).

Acknowledgments: The authors would like to thank the Nanofabrication facility in Suzhou Institute of Nano-tech and Nano-bionics (CAS) for equipment access.

Conflicts of Interest: The authors declare no conflict of interest.

\section{References}

1. Chi, Y.C.; Hsieh, D.H.; Tsai, C.T.; Chen, H.Y.; Kuo, H.C.; Lin, G.R. 450-nm GaN laser diode enables high-speed visible light communication with 9-Gbps QAM-OFDM. Opt. Express 2015, 23, 13051-13059. [CrossRef] [PubMed]

2. Watson, S.; Tan, M.; Najda, S.P.; Perlin, P.; Leszczynski, M.; Targowski, G.; Grzanka, S.; Kelly, A.E. Visible light communications using a directly modulated $422 \mathrm{~nm}$ GaN laser diode. Opt. Lett. 2013, 38, 3792-3794. [CrossRef] [PubMed]

3. Zeller, W.; Naehle, L.; Fuchs, P.; Gerschuetz, F.; Hildebrandt, L.; Koeth, J. DFB Lasers Between 760 nm and 16 $\mu \mathrm{m}$ for Sensing Applications. Sensors 2010, 10, 2492-2510. [CrossRef] [PubMed]

4. Balci, M.H.; Chen, F.; Cunbul, A.B.; Svensen, Ø.; Akram, M.N.; Chen, X. Comparative study of blue laser diode driven cerium-doped single crystal phosphors in application of high-power lighting and display technologies. Opt. Rev. 2018, 25, 166-174. [CrossRef]

5. Shimada, Y.; Chida, Y.; Ohtsubo, N.; Aoki, T.; Takeuchi, M.; Kuga, T.; Torii, Y. A simplified 461-nm laser system using blue laser diodes and a hollow cathode lamp for laser cooling of Sr. Rev. Sci. Instrum. 2013, 84, 063101. [CrossRef]

6. Najda, S.P.; Perlin, P.; Leszczyński, M.; Slight, T.J.; Meredith, W.; Schemmann, M.; Moseley, H.; Woods, J.A.; Valentine, R.; Kalra, S.; et al. A multi-wavelength (uv to visible) laser system for early detection of oral cancer. In Imaging, Manipulation, and Analysis of Biomolecules, Cells, and Tissues XIII; International Society for Optics and Photonics: San Francisco, CA, USA, March 2015; p. 932809.

7. Luo, Y.; Nakano, Y.; Tada, K.; Inoue, T.; Hosomatsu, H.; Iwaoka, H. Fabrication and characteristics of gain-coupled distributed feedback semiconductor lasers with a corrugated active layer. IEEE J. Quantum Electron. 1991, 27, 1724-1731. [CrossRef]

8. Hofstetter, D.; Romano, L.T.; Paoli, T.L.; Bour, D.P.; Kneissl, M. Realization of a complex-coupled InGaN/GaN-based optically pumped multiple-quantum-well distributed-feedback laser. Appl. Phys. Lett. 2000, 76, 2337-2339. [CrossRef]

9. Masui, S.; Tsukayama, K.; Yanamoto, T.; Kozaki, T.; Nagahama, S.I.; Mukai, T. First-order AlInGaN 405 nm distributed feedback laser diodes by current injection. Jpn. J. Appl. Phys. 2006, 45, L749. [CrossRef] 
10. Schweizer, H.; Gräbeldinger, H.; Dumitru, V.; Jetter, M.; Bader, S.; Brüderl, G.; Weimar, A.; \&Härle, V. Laterally coupled InGaN/GaN DFB laser diodes. Phys. Status Solidi A 2002, 192, 301-307. [CrossRef]

11. Hofmann, R.; Gauggel, H.P.; Griesinger, U.A.; Gräbeldinger, H.; Adler, F.; Ernst, P.; Bolay, H.; Harle, V.; Schoz, F.; Schweizer, H.; et al. Realization of optically pumped second-order GaInN-distributed-feedback lasers. Appl. Phys. Lett. 1996, 69, 2068-2070. [CrossRef]

12. Zhang, H.; Cohen, D.A.; Chan, P.; Wong, M.S.; Mehari, S.; Becerra, D.L.; Nakamura, S.; DenBaars, S.P. Continuous-wave operation of a semipolar InGaN distributed-feedback blue laser diode with a first-order indium tin oxide surface grating. Opt. Lett. 2019, 44, 3106-3109. [CrossRef] [PubMed]

13. Holguín-Lerma, J.A.; Ng, T.K.; Ooi, B.S. Narrow-line InGaN/GaN green laser diode with high-order distributed-feedback surface grating. Appl. Phys. Express 2019, 12, 042007. [CrossRef]

14. Masui, S.; Tsukayama, K.; Yanamoto, T.; Kozaki, T.; Nagahama, S.I.; Mukai, T. CW operation of the first-order AlInGaN 405 nm distributed feedback laser diodes. Jpn. J. Appl. Phys. 2006, 45, L1223. [CrossRef]

15. Slight, T.J.; Odedina, O.; Meredith, W.; Docherty, K.E.; Kelly, A.E. InGaN/GaN distributed feedback laser diodes with deeply etched sidewall gratings. IEEE Photonics Technol. Lett. 2016, 28, 2886-2888. [CrossRef]

16. Slight, T.J.; Yadav, A.; Odedina, O.; Meredith, W.; Docherty, K.E.; Rafailov, E.; Kelly, A.E. InGaN/GaN laser diodes with high order notched gratings. IEEE Photonics Technol. Lett. 2017, 29, 2020-2022. [CrossRef]

17. Slight, T.J.; Stanczyk, S.; Watson, S.; Yadav, A.; Grzanka, S.; Rafailov, E.; Perlin, P.; Najda, S.P.; Leszczynski, M.; Gwyn, S.; et al. Continuous-wave operation of (Al, In) GaN distributed-feedback laser diodes with high-order notched gratings. Appl. Phys. Express 2018, 11, 112701. [CrossRef]

18. Kang, J.H.; Martens, M.; Wenzel, H.; Hoffmann, V.; John, W.; Einfeldt, S.; Wernicke, T.; Kneissl, M. Optically pumped DFB lasers based on GaN using 10th-order laterally coupled surface gratings. IEEE Photonics Technol. Lett. 2016, 29, 138-141. [CrossRef]

19. Kang, J.H.; Wenzel, H.; Hoffmann, V.; Freier, E.; Sulmoni, L.; Unger, R.S.; Einfeldt, S.; Wernicke, T.; Kneissl, M. DFB laser diodes based on GaN using 10th order laterally coupled surface gratings. IEEE Photonics Technol. Lett. 2017, 30, 231-234. [CrossRef]

20. Li, J.Z.; Huang, F.; Yang, H.J.; Deng, Z.J.; Liao, M.L. The MOCVD overgrowth studies of III-Nitride on Bragg grating for distributed feedback lasers. In 14th National Conference on Laser Technology and Optoelectronics (LTO 2019); International Society for Optics and Photonics: Shanghai, China, May 2019; p. 111701X.

21. Hofmann, R.; Wagner, V.; Neuner, M.; Off, J.; Scholz, F.; Schweizer, H. Optically pumped GaInN/GaN-DFB lasers: Overgrown lasers and vertical modes. Mater. Sci. Eng. B 1999, 59, 386-389. [CrossRef]

22. Cao, Y.L.; Hu, X.N.; Luo, X.S.; Song, J.F.; Cheng, Y.; Li, C.M.; Liu, C.Y.; Wang, H.; Liow, T.Y.; Lo, G.Q.; et al. Hybrid III-V/silicon laser with laterally coupled Bragg grating. Opt. Express 2015, 23, 8800-8808. [CrossRef]

23. Wenzel, H.; Fricke, J.; Decker, J.; Crump, P.; Erbert, G. High-power distributed feedback lasers with surface gratings: Theory and experiment. IEEE J. Quantum. Elect. 2015, 21, 352-358. [CrossRef]

24. Zolotarev, V.V.; Leshko, A.Y.; Pikhtin, N.A.; Slipchenko, S.O.; Sokolova, Z.N.; Lubyanskiy, Y.V.V.; Voronkova, N.V.; Tarasov, I.Y.S. Integrated high-order surface diffraction gratings for diode lasers. Quantum. Electron. 2015, 45, 1091. [CrossRef]

25. Bao, S.; Song, Q.; Xie, C. The influence of grating shape formation fluctuation on DFB laser diode threshold condition. Opt. Rev. 2018, 25, 330-335. [CrossRef]

26. Crump, P.; Schultz, C.M.; Wenzel, H.; Erbert, G.; Tränkle, G. Efficiency-optimized monolithic frequency stabilization of high-power diode lasers. J. Phys. D Appl. Phys. 2012, 46, 013001. [CrossRef]

(C) 2019 by the authors. Licensee MDPI, Basel, Switzerland. This article is an open access article distributed under the terms and conditions of the Creative Commons Attribution (CC BY) license (http://creativecommons.org/licenses/by/4.0/). 